Article

\title{
Force-Amplified Soft Electromagnetic Actuators
}

\author{
Stanley R. Doerger and Cindy K. Harnett *
}

Electrical and Computer Engineering Department, University of Louisville, Louisville, KY 40208, USA; stanley.doerger@louisville.edu

* Correspondence: c0harn01@louisville.edu; Tel.: +1-502-852-0689

Received: 1 October 2018; Accepted: 23 October 2018; Published: 31 October 2018

\begin{abstract}
Electrically-driven direct current (DC) motors are the core component of conventional robots thanks to the ease of computer control and high torque for their size. However, DC motors are often manually attached and soldered into robotic assemblies, and they are not flexible. For soft robotics, researchers have looked to new, compliant materials that are compatible with 3-D printing or other automated assembly methods. In this work we use a computer-controlled embroidery machine to create flat motor windings in flexible fabrics. We model their electromagnetic fields and present them as linear actuators that move a permanent magnet attached to a cable. The fabrication method puts some constraints on the coil design, which are discussed. However, the planar nature of the embroidered sheets enables the designer to use laminar fabrication methods, such as stacking or layering into parts, during 3-D printing. The soft motor windings produced static holding forces of up to $0.25 \mathrm{~N}$ and could lift a $0.3 \mathrm{~g}$ mass several $\mathrm{cm}$ using direct drive. A 3-D printed mechanical amplifier with two stages was able to quadruple the lifting mass, reducing the travel by a factor of 4 . Machine embroidery-installed cables and motor coils could lead to "bolts and nuts free" fabrication of thin, electrically-driven cable actuators.
\end{abstract}

Keywords: advanced manufacturing; rapid prototyping; electromagnetic actuator; machine embroidery; cable-driven systems

\section{Introduction}

Soft actuators are in demand not only for soft-bodied robots [1,2] and for human-wearable systems [3], but in conventional robotics where a flexible actuator can save space, a soft gripper can add a new function to a robot limb [4], or a new material can cut down on assembly time.

In this work, we develop a flexible, fabric-integrated electromagnetic actuator and demonstrate how to integrate it with cables to transmit forces. Because cable-driven mechanisms offer lightweight yet high-strength actuation, many designs for robotic limb ends have converged on cable-driven actuation [5]. Actuator development efforts split into conventional electromagnetic motors that wind the cable to shorten it, and artificial muscles that pull on cables or even form a segment of the cable themselves. Coiled polymer actuators [6,7], liquid crystal elastomers [8], and shape memory wires are all exciting materials for artificial muscles. These emerging materials integrate well with soft robotics and fabrics [9]. However, most of these systems are thermally driven. They can be controlled by an electronic system, but feedback is generally required to keep the materials at a uniform temperature.

Strong rare-earth magnets have enabled conventional electromagnetic motors to keep pace with newer artificial muscles. While magnets and motor windings are denser than the new polymer actuators, conventional electromagnetic motors offer a high level of control, and for that reason, they have long been used in precision positioning systems including microscope positioning stages, 3-D printers, and more recently thin and flat haptic feedback actuators [10]. By changing the direction or amplitude of a current through a series of wire coils, electromagnetic motors can do "stepping", 
creating well-defined stopping points for the actuator without resorting to a feedback loop. Such digital control over the actuator position is a good match for the microprocessors in robotic systems, and a situation that is harder to find with continuous actuator materials.

Soft electromagnetic actuators are an active research topic because deformable materials are compatible with soft-bodied robots; if the robot's body needs to bend around a corner or flatten to squeeze under a door, the actuator needs to follow suit. Soft robotics researchers have deconstructed the conventional "voice coil," which is an electromagnet made from a current-carrying wire coil that applies a force to a permanent magnet (or sometimes, to a second coil). They have substituted soft materials for permanent magnets in the form of magnetic powder-filled silicone [11-13], made motor housings out of soft silicone to get new types of deformation [12-14], and/or replaced the coil wires with intrinsically stretchable liquid metal filled channels [12,15]. In these examples of soft electromagnetic actuators, an electromagnetic coil pulls a magnet (or another coil) directly along its axis. Applications include vibrotactile motors [12], diaphragm pumps [11], steering and propulsion fins [15], artificial muscle modules that lengthen and shorten [13,14], and actuators that pull on soft levers for gripping objects [12].

In contrast, our actuator passes the magnet along the surface from one coil to another. We increased the actuator's flexibility by making the coil thin and installed it in a soft textile instead of winding it around a rigid spool or printing it on a hard circuit board as in some of the above examples. Although driving this kind of linear actuator is more complex than driving an on-axis electromagnetic actuator, control methods for linear electromagnetic actuators are well developed because of their relevance in valves and other industrial machinery $[16,17]$. They are better suited than on-axis actuators to pulling on cables that require a long $(>5 \mathrm{~mm})$ travel distance.

Planar fabrication is not the usual method for making motors, but as the printed circuit board and semiconductor industries have shown, it has great advantages in making thin devices that integrate quickly into larger structures by lamination. Electromagnets built on soft planar materials, including fabrics, have been explored in art-focused workshops toward wearable displays [18] and audio speakers [19]. Our group [20-22] and others are looking at sewing and embroidery machines as members of the rapid prototyping family that can produce thin and soft electronic antennas [23], sensors, connectors [24], and the actuators described in this work.

\section{Materials and Methods}

\subsection{Fabrication}

Fine copper magnet wire is the building material of electromagnetic motor windings. The wire has an insulating enamel that prevents neighboring coils from shorting out, yet is thin and tough enough to allow dense wire packing. Wire windings create magnetic fields when they carry a current (Figure 1a). We found that 34-gauge $(0.177 \mathrm{~mm}$ diameter) enameled copper wire (Remington Industries, Johnsburg, IL, USA) was compatible with a computerized embroidery machine (Brother PE-770, Brother Industries, Ltd., Nagoya, Aichi, Japan) as bobbin thread (Figure 1b), yet still able to carry currents approaching 1 A without dangerous $\left(>100{ }^{\circ} \mathrm{C}\right)$ heating. Patterns, such as the coils in Figure 1c, were sewn into fabrics, plastic films, and for the experiments discussed in this work, a nonwoven paper-like material (Cut-Away Stabilizer, Sulky of America, Inc., Punta Gorda, FL, USA). Sewing was carried out at 350 stitches per minute, using white polyester embroidery thread to keep the coils in place on the stabilizer.

Where the coil handedness changes from left-handed to right-handed or vice versa, there is a spatial gradient in the magnetic field. A magnet placed on the surface, with its magnetization along the z-direction, will experience the strongest force where the gradient is steepest. It will move toward areas where its magnetic field aligns with the coil field, and away from areas where the magnetic fields are opposed. Coil forces on permanent magnets are the driving principle of conventional direct current (DC) motors, which can work as both rotary and linear actuators. We investigated how to design coils that have steep spatial gradients within the constraints imposed by embroidery. 

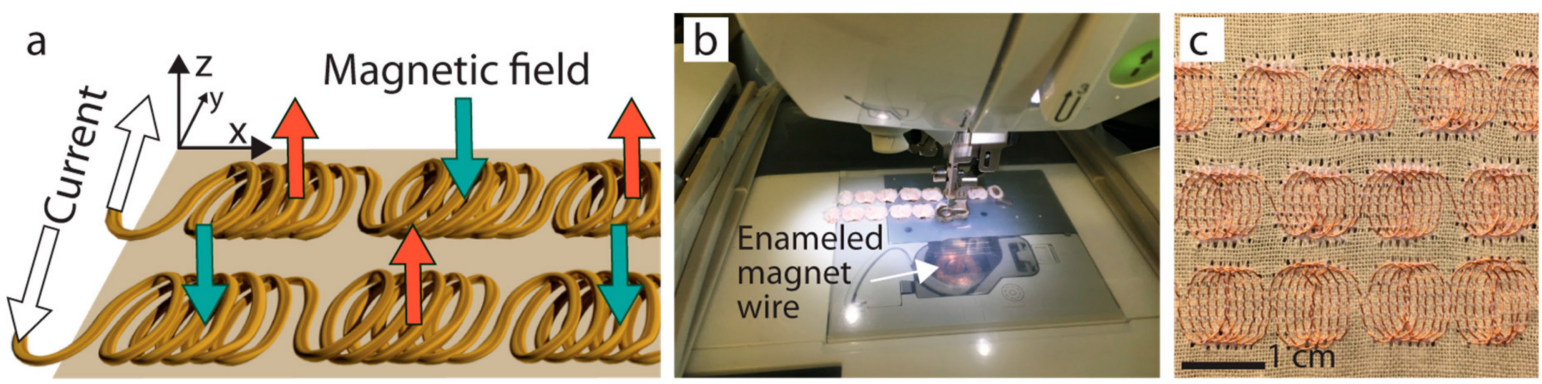

Figure 1. (a) Electric current in flat coils of insulated wire produces magnetic fields. Coils are placed on the surface from right to left and their handedness is changed periodically. (b) Coils were installed in plastic films, papers, and fabrics using a computerized embroidery machine with a bobbin of enamel-insulated magnet wire, sewn with cotton embroidery thread. (c) Coils of fine-gauge magnet wire were patterned into a woven fabric.

\subsection{Design}

Conventional DC motors achieve high torque with hundreds or even thousands of coils. However, an important design consideration from the embroidery machine is that coils need to be thin $(<1 \mathrm{~mm})$, and the needle cannot intersect previously installed wire because it will cut the circuit. A simple, single-layer flat spiral embroiders well (Figure 2a), but its coil number is limited by space-filling. A flattened helix with multiple layers of coils (Figures 1 and $2 b$ ) can produce stronger fields, but as more coils are added, thickness builds up at the edges. The thickness creates an obstacle for the embroidery foot, puts previously sewn wires in the needle path, and stiffens the structure, interfering with applications that require thin and flexible actuators. We designed a third pattern: a flattened square spiral (Figure 2c) that had multiple layers while maintaining uniform thickness with a high fraction of space filling and without stitches (red dots) intersecting previously-installed wire.
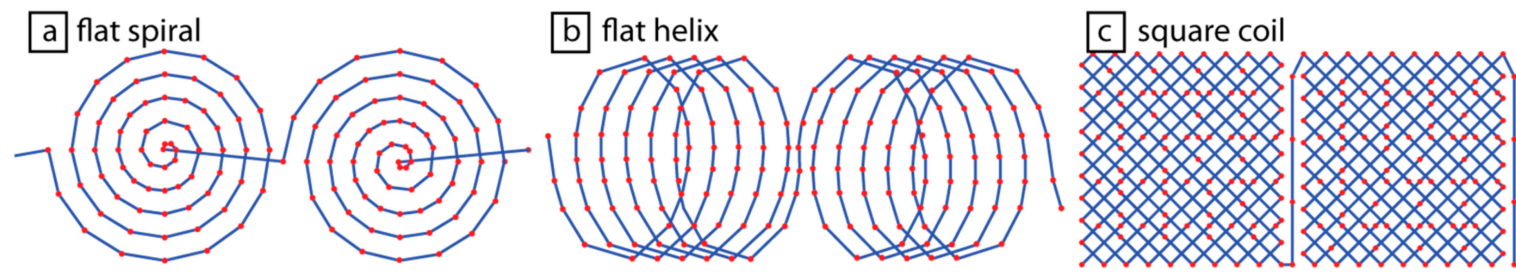

Figure 2. Flat spirals (a), flat helical coils (b), and square coils (c) with alternating handedness.

When designing the square spiral, we considered that the machine's minimum stitch spacing was $0.1 \mathrm{~mm}$. However, the wire diameter is more than $0.1 \mathrm{~mm}$, and because fabrics can stretch, the previously-placed wires can shift because of tension. A larger 0.5-0.6 $\mathrm{mm}$ minimum spacing was more reliable for preventing the needle from intersecting previously-installed wire. We wrote a program [25] with user-adjustable parameters to plot a pair of interleaved, alternating space-filling square coil patterns that do not intersect with future needle locations. Its advantage over the area-filling flat spiral is multiple layers (a maximum of four in this design) versus a single layer for more concentrated magnetic flux. To "print" these patterns, we previously wrote a program that took a list of $x$-y needle coordinates in mm and turned it into a PES (Personal Embroidery System) embroidery file [26].

\subsection{Electromagnetic Modeling}

The force that the coils exert on permanent magnets depends on not only the current in the coils and the strength of the magnet, but on the shape of the coils. When exposed to the coils' magnetic field, the permanent magnets move in a direction that lowers their potential energy $U$ (Equation (1)):

$$
U=-m \cdot B
$$


where $m$ is the magnetic moment of the permanent magnet $\left(\mathrm{A} \cdot \mathrm{m}^{-1}\right)$ and $\boldsymbol{B}$ is the magnetic flux density $(\mathrm{Wb})$ from the coil.

The force $\boldsymbol{F}$ exerted on the magnet is the negative gradient of the potential energy:

$$
\boldsymbol{F}=-\nabla U
$$

A successful coil pattern will produce strong local gradients in the magnetic field over the length scale of the permanent magnet. To compare the force exerted on the same magnet by different coil patterns, we calculated the magnetic fields for each pattern. Coils were modeled in ANSYS Maxwell (ANSYS Inc., Canonsburg, PA, USA) to find the 3-D shape of the fields (Figure S1). However, faster solutions to the force-vs-coil shape problem were obtained using a custom solver made specifically for our two-dimensional (2-D) geometry. Since the magnetic moment is oriented primarily along the z-direction (Figure 1), only $B_{z}$, the z-component of $\boldsymbol{B}$, needed to be solved for finding the potential energy in Equation (1). The magnetic flux density $\boldsymbol{B}$ can be obtained from the magnetic vector potential $A$ :

$$
B=\nabla \times A
$$

where $A$ is calculated from the current $I$ along path $s$ :

$$
A=\frac{\mu_{0} I}{4 \pi} \int \frac{d s}{r}
$$

and where $r$ is the distance from the observer's location to the point along the path of integration. In this work, the current distribution comes from the 2-D coil pattern, described by a path $s\left(x^{\prime}, y^{\prime}\right)$, multiplied by the coil current $I$ :

$$
A(x, y)=\frac{\mu_{0} I}{4 \pi} \int \frac{d s}{\sqrt{\left(x^{\prime}-x\right)^{2}+\left(y^{\prime}-y\right)^{2}}}
$$

where the magnetic permeability $\mu_{0}=4 \pi \times 10^{-7} \mathrm{H} / \mathrm{m}$ and the path differential $d s$ can be written as $d x^{\prime} \hat{x}+d y^{\prime} \hat{y}$ for the 2-D embroidered coil paths.

Since the path is always encoded for embroidery patterns as a sequence of straight line segments, such as those in Figure 2, the segments can each be parameterized in $x^{\prime}$, with $y^{\prime}=m_{i} x^{\prime}+b_{i}$, where $m_{i}$ and $b_{i}$ are constants for the $i$ th segment, and where $x$ runs from $x_{i 0}$ to $x_{i 1}$. The contribution of the $i$ th segment to $A$ is then:

$$
A_{i}(x, y)=\frac{\mu_{0} I}{4 \pi} \int_{x_{i 0}}^{x_{i 1}} \frac{d x^{\prime}\left(\hat{x}+m_{i} \hat{y}\right)}{\sqrt{\left(x^{\prime}-x\right)^{2}+\left(m_{i} x^{\prime}+b_{i}-y\right)^{2}}}
$$

which has both $x$ - and $y$ - components. The exception is the case where a segment runs purely along the $y$-direction. For these vertical segments, which run from $y_{i 0}$ to $y_{i 1}$, the contribution of the $i$ th segment to $A$ is:

$$
A_{i}(x, y)=\frac{\mu_{0} I}{4 \pi} \int_{y_{i 0}}^{y_{i 1}} \frac{d y^{\prime} \hat{y}}{\sqrt{\left(x_{i}-x\right)^{2}+\left(y^{\prime}-y\right)^{2}}}
$$

The total magnetic vector potential $A$ at location $(x, y)$ is obtained by summing over the $N$ embroidered segments:

$$
A(x, y)=\sum_{i=0}^{N} A_{i}(x, y)
$$


To find just $B_{z}$, the z-component of the curl of $A$ is calculated by taking derivatives with respect to the non-primed coordinates in Equations (6) and (7):

$$
B_{z}=\frac{d A_{y}}{d x}-\frac{d A_{x}}{d y}
$$

Analytic solutions exist for each of the resulting integrals in Equation (9), eliminating numerical integration and making the solver run considerably faster than the general 3-D case. Using the result for $B_{z}$, the potential energy $U$ is computed using Equation (1) with the magnetic dipole moment oriented along $\hat{z}$. Because the permanent magnet samples have different locations with different magnetic fields, the magnet is treated as a grid of point dipoles $m$, and the total potential energy is:

$$
U=-m \sum_{i} B_{z}\left(x_{i}, y_{i}\right)
$$

where $x_{i}$ and $y_{i}$ denote the location of the $i$ th dipole in the magnet.

Potential energy at the centroid of the magnet can be plotted as a landscape to compare different designs (Figure 3), and the net force on the magnet can calculated using Equation (1) on $U$ :

$$
F_{x}=-\frac{d U}{d x}, F_{y}=-\frac{d U}{d y}
$$

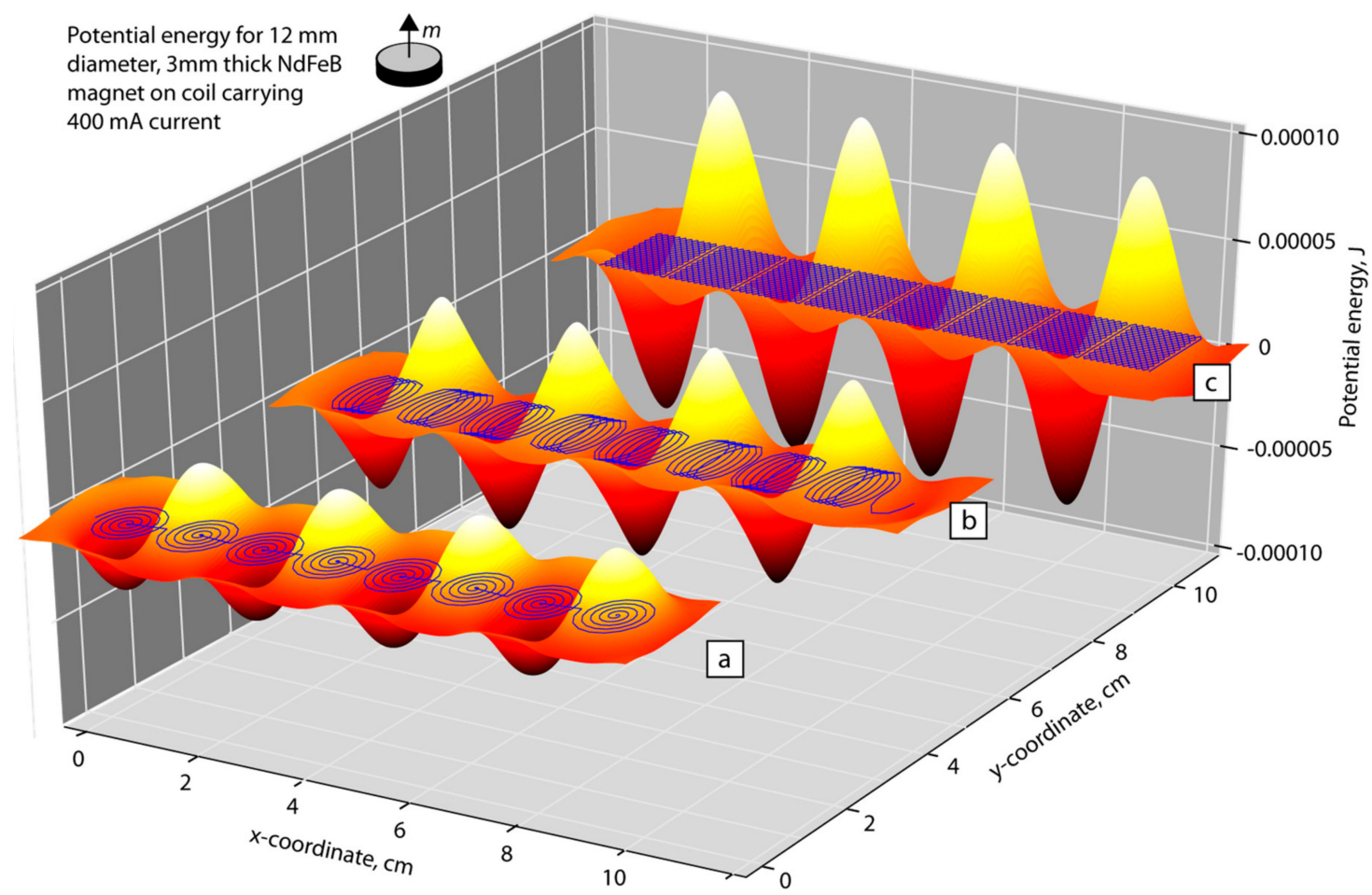

Figure 3. Potential energy landscapes for the three designs in Figure 2: (a) flat spiral, (b) flat helix, and (c) square coil. Because the square coil packs more overlapping loops into the same area as the other two designs, it generates a higher maximum magnetic field and higher potential energy for the same magnet.

In the coordinate system of Figure $1 \mathrm{a}, F_{x}$ determines how much force the magnet can exert on a cable it is driving, while $F_{y}$ describes sideways forces that determine whether the magnet stays on the path. This analysis ignores the thickness of the magnet, which in our work is typically a thin disc or tile; however, the code includes a z-offset to prevent the denominator in Equations (6) and (7) 
from becoming 0 when a dipole coincides with a wire location. For example, Equation (6) is modified as follows:

$$
A_{i}(x, y)=\frac{\mu_{0} I}{4 \pi} \int_{y_{i 0}}^{y_{i 1}} \frac{d y^{\prime} \hat{y}}{\sqrt{\left(x_{i}-x\right)^{2}+\left(y^{\prime}-y\right)^{2}+z_{o}^{2}}}
$$

The offset $z_{0}$ represents the thickness of the enamel coating that prevents the magnet from touching the wire surface. It may also be adjusted to account for contributions to $U$ from higher layers in a thicker magnet. The code that calculates the potential energy landscape from a given coil path is available online at Reference [27].

\subsection{Driving Circuit}

A permanent magnet set on one of the coil patterns in Figure 3 will "fall" downhill on the potential energy diagram, causing it to move along the ribbon. However, when it falls into a low potential energy location where the magnet and coil fields align, it will stop. We switched the current direction through two sets of interleaved coils to keep the potential energy landscape (and therefore the magnet) moving. The second coil was offset by one-half the coil pitch. By switching on the second coil, a potential energy well became a hill. The magnet, suddenly on the positive- $x$ side of the hill, was attracted to the new potential well ahead of it. For each coil, the direction of a $400 \mathrm{~mA}$ DC current was independently switched using a computer at frequencies around $5 \mathrm{~Hz}$. With coil resistances of approximately $3 \Omega$, a commercial motor driver board (Supplementary Information) was used to handle the currents and inductive loads of the coil circuit. Figure 4 shows the interleaved coil pair along with simulation results for the net force along the $x$-direction during each step of the four-step driving cycle.
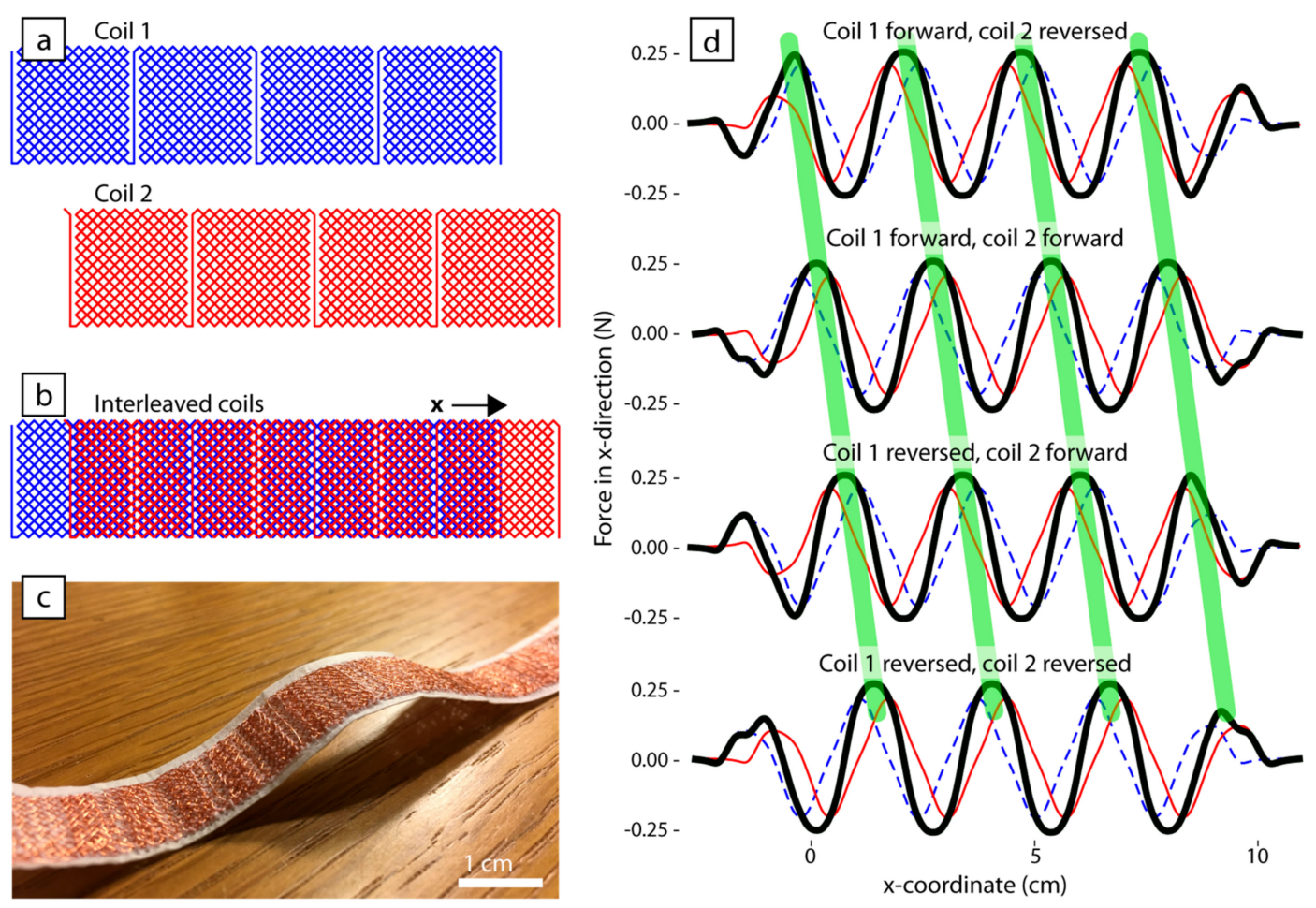

Figure 4. (a) A pair of square coils was offset by half the coil pitch (b) and interleaved. (c) The embroidered square coil pattern had both sets of coils stitched into the same layer, offset sufficiently to prevent the needle from intersecting the wire. (d) The coil pair was driven in a four-step sequence to create continuous motion along the x-direction. Green lines track the maximum force exerted on a magnet at each of the four steps of the cycle. During any step, a magnet at the maximum-force position will move in the $+x$-direction until it experiences zero force. In the next step, the old zero-force location is the new maximum force location. 
The three designs from Figure 2 were simulated in the interleaved format of Figure 4 . Table 1 lists the calculated maximum $x$-force on a $12 \mathrm{~mm}$ diameter, $3 \mathrm{~mm}$ thick disc-shaped NdFeB magnet magnetized along its z-axis, with each coil carrying a current of $\pm 400 \mathrm{~mA}$. The calculations showed that the square coils could produce nearly three times the force of the flat spirals.

Table 1. Simulated $x$-force from interleaved versions of each of the three designs in Figure 2.

\begin{tabular}{ccc}
\hline Design & Wire Length Per Coil $(\mathbf{c m})$ & Maximum \\
\hline Flat spirals (Figure 2a) & 10.2 & 9.8 \\
Flat helix (Figure 2b) & 16.9 & 17.0 \\
Square coils (Figure 2c) & 37.0 & 26.5 \\
\hline
\end{tabular}

\subsection{Force Measurements}

For testing the holding strength of the potential energy wells, a DC power supply was used to maintain a constant current at levels between 0 and $1 \mathrm{~A}$ while forces were applied to the magnet using the method of Figure 5. Magnets were tested in vertical stacks, and in horizontal chains with alternating magnet orientation.
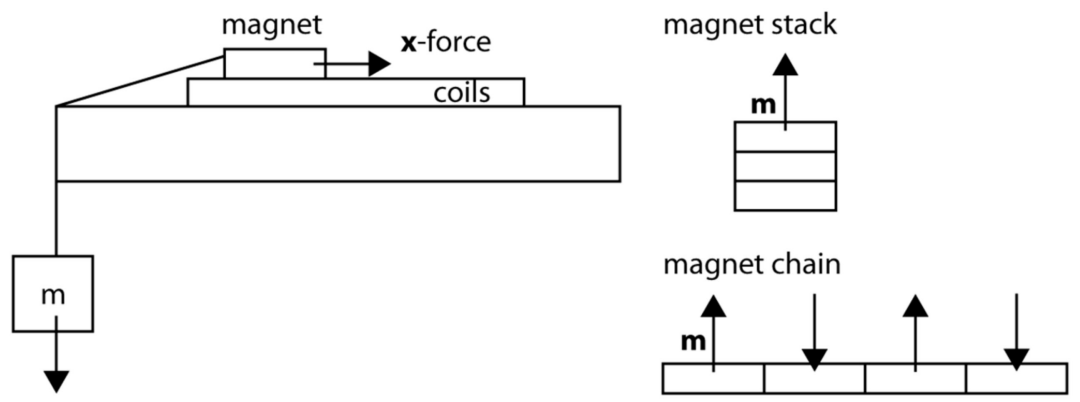

Figure 5. Force testing. A paper weighing basket $(0.1 \mathrm{~g})$ was attached to the magnet using a string and suspended over the edge of the table. While the coils were energized, weights were added to the basket until the magnet began to slip.

\subsection{Mechanical Amplification}

Because cable-driven actuation often requires a high force but only a short travel distance, we investigated a mechanical amplifier using small 3-D printed compound sliders (Figure 6) that trade distance for force. Sliders were attached to the magnets using cotton sewing thread and tape, and were inserted on the table between the actuator and the hanging mass.

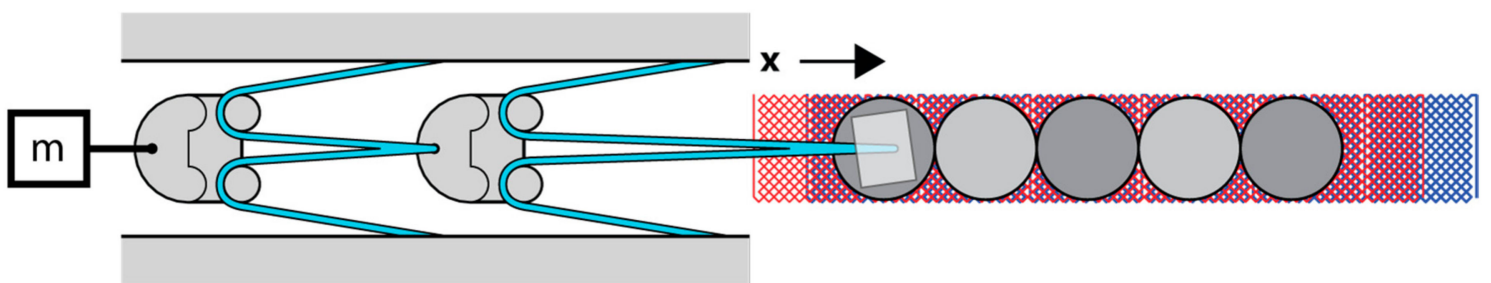

Figure 6. Mechanical amplification using two sliders. Each slider in the series doubled the force lifting the mass (minus friction), and halved the distance traveled by the cable.

\section{Results}

\subsection{Force Measurements}

Force measurements were collected using the DC current method to determine how much force the square coil design in Figure $4 \mathrm{~b}$ could hold for different magnet configurations at a fixed current 
(Figure 7a) and for the five-magnet chain with different amounts of current (Figure 7b). All magnets were the $12 \mathrm{~mm}$ diameter, $3 \mathrm{~mm}$ thick $\mathrm{NdFeB}$ discs described previously.
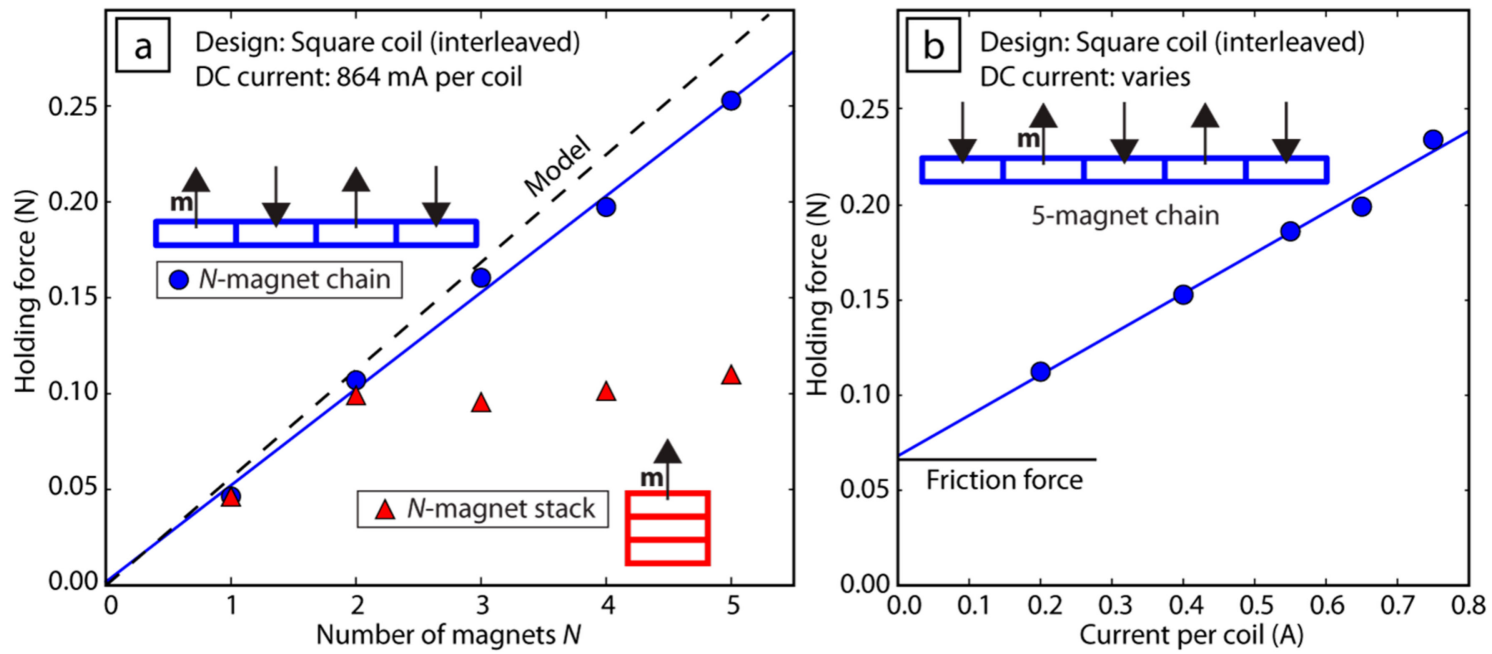

Figure 7. Experimental results from the square coils. (a) Holding force vs magnet number for chain and stack configurations at a fixed current. (b) Holding force vs current for a five-magnet chain.

The model from Section 2.3 produced a maximum force on a single magnet of $26.5 \mathrm{mN}$ (Table 1 and Figure $4 \mathrm{~d}$ ) when two square coils were interleaved and fed $400 \mathrm{~mA}$. This result was scaled by $(864 / 400)$ to account for the $864 \mathrm{~mA}$ current per coil in Figure 7a, and plotted as a line running through the origin (dashed line on Figure 7a). Magnet chains showed a linear relationship between chain length $\mathrm{N}$ and force (solid blue line on Figure 7a), while stacking the magnets only increased the force for $N=2$. When the five-magnet chain was tested at varying currents (Figure $7 \mathrm{~b}$ ), the holding force was linear in current and did not extrapolate to the origin when the current was off, instead intercepting at $0.068 \mathrm{~N}$. With currents in the 800-900 mA range for these experiments, surface temperatures of $40{ }^{\circ} \mathrm{C}$ were measured. Because high currents could damage the magnet wire, we kept the current below $1 \mathrm{~A}$ in the static tests. In the dynamic tests below, current-limiting resistors in series with the coils ensured that the current stayed below $1 \mathrm{~A}$ for any length of coil.

\subsection{Force Amplification}

Dynamic tests were carried out using the setup in Figure S2 to produce cyclic motion of the five-magnet chain on the square coil design. Because the cyclic motion system was powered using a Universal Serial Bus (USB) port with a current limit of $500 \mathrm{~mA}$, the tests were conducted with $400 \mathrm{~mA}$ per coil instead of the higher currents available from the DC power supply. The force amplifier in Figure 6 was connected with no sliders, one, or two sliders, and weights in $0.18 \mathrm{~g}$ increments were added until the magnet chain would no longer move forward. The maximum mass the system could lift is listed in Table 2.

Table 2. Measured mass that could be lifted by the square coil at $400 \mathrm{~mA}$ using a five-magnet chain with the force amplifier.

\begin{tabular}{ccc}
\hline Number of Sliders in the Amplifier & Maximum Mass Lifted (g) & Corresponding Force (mN) \\
\hline 0 (Direct drive) & 0.37 & 3.7 \\
1 & 0.74 & 7.3 \\
2 & 1.49 & 14.7 \\
\hline
\end{tabular}




\section{Discussion}

Results showed that the model could predict the forces exerted by the square coil to within $10 \%$, and suggested some methods for increasing the force. In Figure $7 \mathrm{a}$, magnet stacking did not improve the performance as much as making a flat, low chain because of the rapid drop-off of the magnetic field with height (Figure S1). Thin magnets assembled into a chain naturally when placed in the alternating magnetization pattern because they could form closed magnetic flux loops with their neighbors. These chains had some mechanical flexibility and provided a straightforward way to scale up the force for a given current. Coils on top would double the force, and a parallel track run 180 degrees out of phase could add more force as well as keep the magnet array centered.

The model in Figure 7 a overpredicted the force by about $10 \%$ at the high end of the range. As the magnet chain got heavier, it exerted more force but was also be subject to more friction force. If the exerted force continued to scale linearly with chain length, the effect cancelled out, so friction was not likely to be the source of the growing discrepancy at larger $N$. However, these magnets at $12 \mathrm{~mm}$ diameter were slightly smaller than the coil pitch of $13 \mathrm{~mm}$, meaning that as the chain grew longer, not every magnet could align perfectly with a potential energy well. Figure 7a may be showing the size mismatch drop-off at $N=4$ and $N=5$.

In Figure $7 \mathrm{~b}$, increasing the current caused the force to grow linearly as predicted by the model. The y-intercept at $0.068 \mathrm{~N}$ was likely to have originated from friction, because even when the current was off, a weight like the five-magnet chain (mass $m=13.8 \mathrm{~g}$ ) took some force to move. The force may be estimated as $\mu m g$, where $\mu$ is the coefficient of friction (typically 0.5 for metals on plastics [28]) and $g$ is gravitational acceleration of $9.8 \mathrm{~m} \cdot \mathrm{s}^{-2}$; the result was $0.0676 \mathrm{~N}$.

The dynamic tests showed that forces could be nearly quadrupled in the two-stage force amplifier, despite friction between the thread and 3-D-printed sliders that were not real pulleys. The system was assembled by hand, which was counter to our goals of an easy to assemble system. However, we have previously demonstrated how an embroidery machine can install fishing-line actuation cables in a 3-D printed part during assembly $[29,30]$. Short, local cables, such as the cables running through each slider, are compatible with this method and are rarely considered for designs because they are so labor intensive. With the versatile set of materials that work with embroidery, there is potential for an amplifier, cables, and a motor to be installed in the same thin sheet for placement during 3-D printing.

Supplementary Materials: The following are available online at https:/ / www.dropbox.com/s/wnihfyy42k652b2/ supporting \%20information \%20for\%20planar\%20fabrication $\% 20$ of $\% 20$ soft $\% 20 \mathrm{em} \% 20$ actuators.docx? dl=0. S1: Magnetic field isosurface plot, S2: Driving circuit, and S3: Links to videos of actuation and amplification.

Author Contributions: Conceptualization, C.K.H.; methodology, S.R.D. and C.K.H.; software, C.K.H.; validation, C.K.H.; formal analysis, S.R.D. and C.K.H.; investigation, S.R.D. and C.K.H., resources, C.K.H.; data curation, S.R.D. and C.K.H.; writing-original draft preparation, S.R.D. and C.K.H., writing-review and editing, C.K.H.; visualization, S.R.D. and C.K.H.; supervision, C.K.H.; project administration, C.K.H.; funding acquisition, C.K.H.

Funding: This work was supported by the Kentucky Science and Engineering Foundation through grant KSEF-3503-RDE-01.

Acknowledgments: J. Paik and K. O’Brien for discussions, and Z. Johnson for coil rendering in Figure 1 a.

Conflicts of Interest: The authors have no conflict of interest.

\section{References}

1. Christianson, C.; Goldberg, N.N.; Deheyn, D.D.; Cai, S.; Tolley, M.T. Translucent soft robots driven by frameless fluid electrode dielectric elastomer actuators. Sci. Robot. 2018, 3, eaat1893. [CrossRef]

2. Shepherd, R.F.; Stokes, A.A.; Freake, J.; Barber, J.; Snyder, P.W.; Mazzeo, A.D.; Cademartiri, L.; Morin, S.A.; Whitesides, G.M. Using explosions to power a soft robot. Angew. Chem. Int. Ed. 2013, 52, 2892-2896. [CrossRef] [PubMed] 
3. Lee, S.; Karavas, N.; Quinlivan, B.T.; Ryan, D.L.; Perry, D.; Eckert-Erdheim, A.; Murphy, P.; Goldy, T.G.; Menard, N.; Athanassiu, M.; et al. Autonomous Multi-Joint Soft Exosuit for Assistance with Walking Overground. In Proceedings of the 2018 IEEE International Conference on Robotics and Automation (ICRA), Brisbane, Australia, 21-25 May 2018.

4. Brown, E.; Rodenberg, N.; Amend, J.; Mozeika, A.; Steltz, E.; Zakin, M.R.; Lipson, H.; Jaeger, H.M. Universal robotic gripper based on the jamming of granular material. Proc. Natl. Acad. Sci. USA 2010, 107, 18809-18814. [CrossRef]

5. Carrozza, M.C.; Suppo, C.; Sebastiani, F.; Massa, B.; Vecchi, F.; Lazzarini, R.; Cutkosky, M.R.; Dario, P. The SPRING hand: Development of a self-adaptive prosthesis for restoring natural grasping. Auton. Robots 2004, 16, 125-141. [CrossRef]

6. Haines, C.S.; Lima, M.D.; Li, N.; Spinks, G.M.; Foroughi, J.; Madden, J.D.; Kim, S.H.; Fang, S.; de Andrade, M.J.; Göktepe, F.; Göktepe, Ö. Artificial muscles from fishing line and sewing thread. Science 2014, 343, 868-872. [CrossRef] [PubMed]

7. Yip, M.C.; Niemeyer, G. High-performance robotic muscles from conductive nylon sewing thread. In Proceedings of the 2015 IEEE International Conference on Robotics and Automation (ICRA), Seattle, WA, USA, 20-30 May 2015; pp. 2313-2318.

8. He, Q.; Wang, Z.; Song, Z.; Cai, S. Bioinspired Design of Vascular Artificial Muscle. Adv. Mater. Technol. 2018, 1800244. [CrossRef]

9. Chenal, T.P.; Case, J.C.; Paik, J.; Kramer, R.K. Variable stiffness fabrics with embedded shape memory materials for wearable applications. In Proceedings of the 2014 IEEE/RSJ International Conference on Intelligent Robots and Systems (IROS 2014), Chicago, IL, USA, 14-18 September 2014; pp. 2827-2831.

10. Salerno, M.; Firouzeh, A.; Paik, J. A low profile electromagnetic actuator design and model for an origami parallel platform. J. Mech. Robot. 2017, 9, 041005. [CrossRef]

11. Yamahata, C.; Lotto, C.; Al-Assaf, E.; Gijs, M.A.M. A PMMA valveless micropump using electromagnetic actuation. Microfluid. Nanofluid. 2005, 1, 197-207. [CrossRef]

12. Do, T.N.; Phan, H.; Nguyen, T.Q.; Visell, Y. Miniature Soft Electromagnetic Actuators for Robotic Applications. Adv. Funct. Mater. 2018, 28, 1800244. [CrossRef]

13. Ebrahimi, N.; Nugroho, S.; Taha, A.F.; Gatsis, N.; Gao, W.; Jafari, A. Dynamic Actuator Selection and Robust State-Feedback Control of Networked Soft Actuators. arXiv, 2018; arXiv:1804.01615.

14. Nemitz, M.P.; Mihaylov, P.; Barraclough, T.W.; Ross, D.; Stokes, A.A. Using voice coils to actuate modular soft robots: Wormbot, an example. Soft Robot. 2016, 3, 198-204. [CrossRef] [PubMed]

15. Guo, R.; Sheng, L.; Gong, H.; Liu, J. Liquid metal spiral coil enabled soft electromagnetic actuator. Sci. China Technol. Sci. 2018, 61, 516-521. [CrossRef]

16. Mercorelli, P.; Lehmann, K.; Liu, S. Robust Flatness Based Control of an Electromagnetic Linear Actuator Using Adaptive PID Controller. In Proceedings of the 42nd IEEE Conference on Decision and Control, Maui, HI, USA, 9-12 December 2003.

17. Chen, L.; Mercorelli, P.; Liu, S. A Kalman estimator for detecting repetitive disturbances. In Proceedings of the 2005 American Control Conference, Portland, OR, USA, 8-10 June 2005; pp. 1631-1636.

18. Flip-Dot Actuator on Fabric. Available online: https://www.kobakant.at/DIY/?p=5915 (accessed on 28 September 2018).

19. Soft Speaker on Fabric. Available online: https://blog.adafruit.com/2015/04/01/machine-embroideredspeaker-wearablewednesday / (accessed on 28 September 2018).

20. Kimmer, C.J.; Harnett, C.K. Combining Strings and Fibers with Additive Manufacturing Designs. In Proceedings of the ASME 2016 International Design Engineering Technical Conferences and Computers and Information in Engineering Conference, Charlotte, NC, USA, 21-24 August 2016; p. V004T05A014.

21. Harnett, C.K.; Zhao, H.; Shepherd, R.F. Stretchable Optical Fibers: Threads for Strain-Sensitive Textiles. Adv. Mater. Technol. 2017, 2, 1700087. [CrossRef]

22. Ceron, S.; Cohen, I.; Shepherd, R.; Pikul, J.; Harnett, C. Fiber Embroidery of Self-Sensing Soft Actuators. Biomimetics 2018, 3, 24. [CrossRef]

23. Kiourti, A.; Lee, C.; Volakis, J.L. Fabrication of textile antennas and circuits with $0.1 \mathrm{~mm}$ precision. IEEE Antennas Wirel. Propag. Lett. 2016, 15, 151-153. [CrossRef]

24. Rahimi, R.; Ochoa, M.; Yu, W.; Ziaie, B. A sewing-enabled stitch-and-transfer method for robust, ultra-stretchable, conductive interconnects. J. Micromech. Microeng. 2014, 24, 095018. [CrossRef] 
25. Python Notebook for Plotting Square Coils. Available online: https://github.com/harnettlab/ iPythonNotebookRepo/blob/master/FiberDrive/SquareCoil.ipynb (accessed on 23 September 2018).

26. PESWrite Code for Making PES Embroidery Files from Coordinates. Available online: https://github.com/ harnettlab/iPythonNotebookRepo/blob/master / PESWrite2.ipynb (accessed on 23 September 2018).

27. Python Notebook for Force Simulation, and Three Example Coil Patterns. Available online: https://github. com/harnettlab/iPythonNotebookRepo/tree/master/FiberDrive (accessed on 23 September 2018).

28. Friction Coefficient Table. Available online: http://www.engineershandbook.com/Tables/ frictioncoefficients.htm (accessed on 28 September 2018).

29. Harnett, C.K.; Wagner, B.P. Expanding the Robotics Materials Set with Machine Embroidery. In Proceedings of the Material Robotics-Bridging Materials Science and Robotics Workshop at the Robotics: Science and Systems (RSS), Cambridge, MA, USA, 15 July 2017.

30. Harnett, C.K.; Wagner, B.P. Integrating Fibers in Robotics Using Automation. Module of Online Soft Robotics Toolkit. Available online: https://softroboticstoolkit.com/integrating-fibers (accessed on 23 September 2018).

(C) 2018 by the authors. Licensee MDPI, Basel, Switzerland. This article is an open access article distributed under the terms and conditions of the Creative Commons Attribution (CC BY) license (http://creativecommons.org/licenses/by/4.0/). 